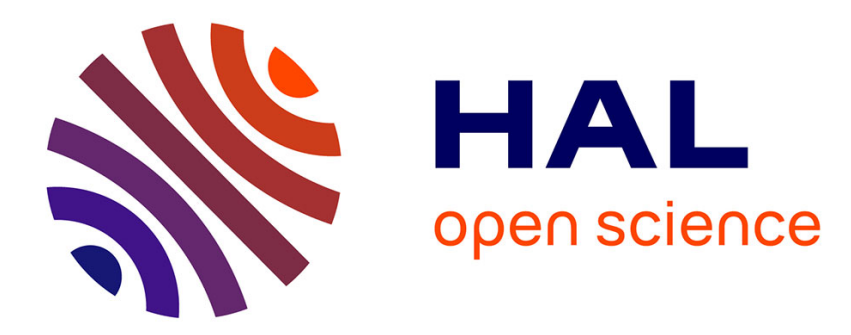

\title{
An Empirical Method of Dispersion Correction in the Compressive Hopkinson Bar Test \\ D. Gorham, X. Wu
}

\section{To cite this version:}

D. Gorham, X. Wu. An Empirical Method of Dispersion Correction in the Compressive Hopkinson Bar Test. Journal de Physique IV Proceedings, 1997, 07 (C3), pp.C3-223-C3-228. 10.1051/jp4:1997340 . jpa-00255497

\section{HAL Id: jpa-00255497 https://hal.science/jpa-00255497}

Submitted on 1 Jan 1997

HAL is a multi-disciplinary open access archive for the deposit and dissemination of scientific research documents, whether they are published or not. The documents may come from teaching and research institutions in France or abroad, or from public or private research centers.
L'archive ouverte pluridisciplinaire HAL, est destinée au dépôt et à la diffusion de documents scientifiques de niveau recherche, publiés ou non, émanant des établissements d'enseignement et de recherche français ou étrangers, des laboratoires publics ou privés. 


\title{
An Empirical Method of Dispersion Correction in the Compressive Hopkinson Bar Test
}

\author{
D.A. Gorham and X.J. Wu \\ Faculty of Technology, The Open University, Walton Hall, Milton Keynes, MK7 6AA, U.K.
}

\begin{abstract}
The dispersion of longitudinal stress waves as they propagate in pressure bars distorts the shape of the pulse, and so can be a limitation to the accuracy of high strain rate tests such as the compressive split Hopkinson pressure bar (SHPB). The method of dispersion correction described in this paper is based on a bar phase characteristic which is derived entirely from measured stress pulses generated by the elastic impact of small spheres. This method does not depend on any theoretical model of wave propagation, and automatically includes all distortions that could arise in mechanical or electronic aspects of the test. It is quick and convenient enough to be used on a routine basis to improve the accuracy of SHPB tests.
\end{abstract}

Résumé : La dispersion des ondes de contrainte longitudinales transportées dans des barres déforme l'impulsion, ce qui peut restreindre la précision des essais à grande vitesse de déformation, tels que ceux réalisés à la barre d'Hopkinson en compression. Les modalités de la correction de la dispersion décrites dans le présent article sont fondées sur des caractéristiques de phases dérivếes entièrement d'impulsions mesurées, crées par l'impact élastique de petites sphères. Cette méthode ne dépend d'aucun modele théorique de propagation d'ondes, et prend automatiquement en compte toutes les erreurs possibles dues aux aspects mécaniques ou électroniques de l'essai. Elle est suffisamment rapide et facile pour êue utilisée régulièrement afin d'améliorer la précision des essais à la barre d'Hopkinson.

\section{INTRODUCTION}

In the compressive version of the split Hopkinson pressure bar (SHPB), measurements of stress pulses at some distance along the bars are used to determine conditions at the specimen interfaces, and hence derive a stress-strain history using the theory of one-dimensional elastic wave propagation [1]. The propagation of longitudinal stress waves is, however, dispersive for wavelengths which are of the same order of magnitude as the bar diameter. This causes distortion of short stress pulses, and hence the signals registered by the gauges in both bars are not necessarily an accurate indication of conditions at the specimen boundary. With a given bar diameter, this limitation to the accuracy with which specimen stress and strain can be measured becomes more important as the strain rate, and hence the high frequency content of the signals, increases.

However, since the dispersive distortion is created by the spread of phase velocities over the signal spectrum, it should in principle be possible to reverse the effects of propagation by applying appropriate phase shifts to each frequency component. Several authors, including Hsieh and Kolsky [2], Gorham [3], Follansbee and Frantz [4], Gong et al. [5], Gary et al. [6], Safford [7], Lee and Crawford [8], and Lifshitz and Leber [9], have carried out this procedure using phase corrections derived from the theoretical analysis of wave propagation in bars. This technique is successful for moderately dispersed signals, but becomes inaccurate for large amounts of dispersion. The inaccuracies arise largely because of the differences between the theoretical and experimental conditions, such as end effects and the distribution of loading, and errors in the empirically determined material constants.

Direct measurement of the phase characteristics of the bar under the conditions in which it is to be used avoids these inaccuracies, and is in principle a more reliable basis for correction. Lee $e t$ al [10] have used a comparatively complex time of arrival analysis of shock signals to derive an empirical dispersion relation for a bar, and demonstrated an improved accuracy of correction over the theoretical methods of Gorham [3] and others. Gorham and Wu [11] have described a simpler method for deriving a dispersion characteristic from measurements of elastic impact stress from small spheres, and have applied it to the correction of signals from larger particle impacts. This method is a direct measurement of phase that makes no theoretical assumptions about the nature of the wave propagation. The present paper describes the application of this empirical method to the SHPB, and demonstrates a considerable improvement over earlier techniques based on theoretical models. 


\section{THE IDEAL PULSE FROM THE ELASTIC IMPACT OF SPHERES}

In our method, the phase characteristic is found from measurements of the elastic impact of small spheres on the end of the bar. Classical Hertzian theory predicts that the force history arising from an elastic collision between a sphere and a flat is approximately of cosine shape, as sketched in Figure 1. The magnitude and phase spectra obtained from a Fast Fourier Transform (FFT) operation on this ideal form of signal are shown in Figure 2, where the frequency axis is marked in units of component number. Only the first 200 points are shown here, each point representing a frequency interval of $9.765 \mathrm{kHz}$, and so giving it maximum abscissa value of $1.953 \mathrm{MHz}$. Notice that the magnitude spectrum in the figure displays a serics of well defined minima, while the phase consists of a series of $\pi$ steps which correspond to these magnitude minima.

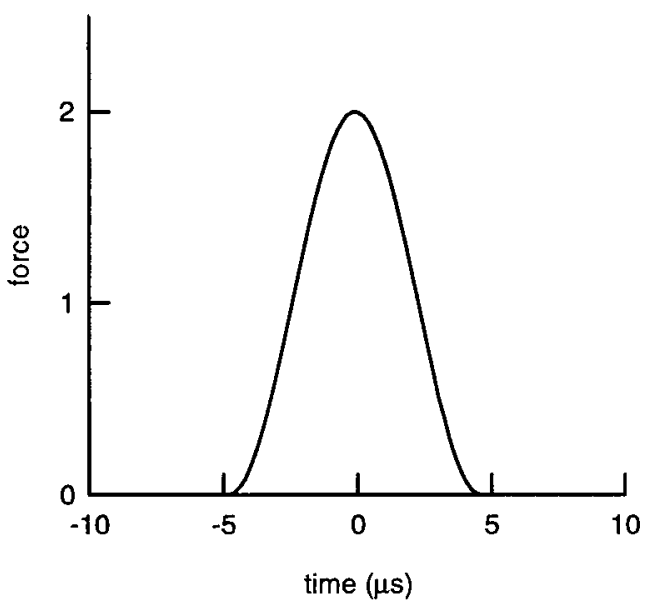

Figure 1: Elastic impact force history of a sphere on a plate, according the Hertzian theory.
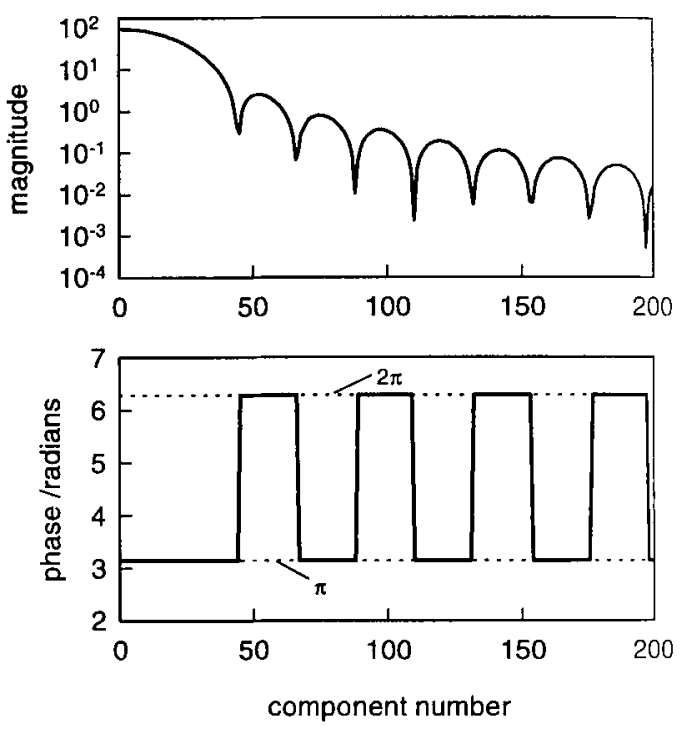

Figure 2: Phase and magnitude spectra for the signal of Figure 1.

This simple variation of phase arises from the symmetry of the waveform about the time origin. Groups of frequency components between the steps add up in phase, to create a symmetrical sum with either a positive going maximum at time zero $(\phi=\pi)$ or a negative going maximum $(\phi=0$ or $2 \pi)$. Any phase value which is not a multiple of $\pi$ would not contribute to a symmetrical waveform. The exact shape of the pulse, which is the total of all these contributing sums, is then determined by the positions at which the $\pi$ steps occur.

If the collision involves plastic deformation, then the stress pulse may be slightly asymmetric. A similar phase spectrum is found in this case, but the phase values may vary a little between the steps (Gorham \& Wu [11]). These simple forms of phase spectra found with ideal pulses and their link to the magnitude spectrum provide the key to empirical correction of experimental signals.

\section{STRESS PULSE MEASUREMENTS}

The technique for dispersion correction will be illustrated by a $6.7 \mathrm{~mm}$ diameter SHPB system, using Ti6Al4V bars instrumented with unbacked, silicon strain gauges located approximately $100 \mathrm{~mm}$ from the specimen ends. The gauges had a physical length of $1 \mathrm{~mm}$, a gauge length of $0.5 \mathrm{~mm}$ and a width of 0.15 $\mathrm{mm}$. The rise time of their response, about $0.1 \mu \mathrm{s}$, is fast enough not to create a bandwidth limitation for the signals measured in this work. Two gauges were mounted on opposite sides of the bar, and connected in series so that unsymmetrical bending waves would be cancelled out. The gauges were energised from a constant d.c. voltage source in series with a resistance. The signal was acquired with an 8-bit $200 \mathrm{MS} \mathrm{s}^{-1}$ digital recorder, and data was transferred to a PC for processing with Matlab analysis software.

Measurement of impact force signals was carried out with the bars mounted vertically. Steel spheres in the diameter range of $0.6 \mathrm{~mm}$ to $4 \mathrm{~mm}$ were dropped from a height of less than $0.5 \mathrm{~m}$ onto the centre of the end face of the bar. Collision velocities were less than $2 \mathrm{~ms}^{-1}$, low enough for impacts to be largely elastic and hence the pulses to be almost symmetrical. 
Two typical examples of these signals are shown in Figure 3. The larger size ( $4 \mathrm{~mm}$ ) shows comparatively small effects of dispersion, with only a small oscillatory overshoot after the main pulse. However, the smaller size $(0.6 \mathrm{~mm})$ is dominated by its effects, with large oscillations carrying on for a long time after a barely discernible main pulse. Note that the time origin in the measured traces has been shifted to the centre of the main pulse.
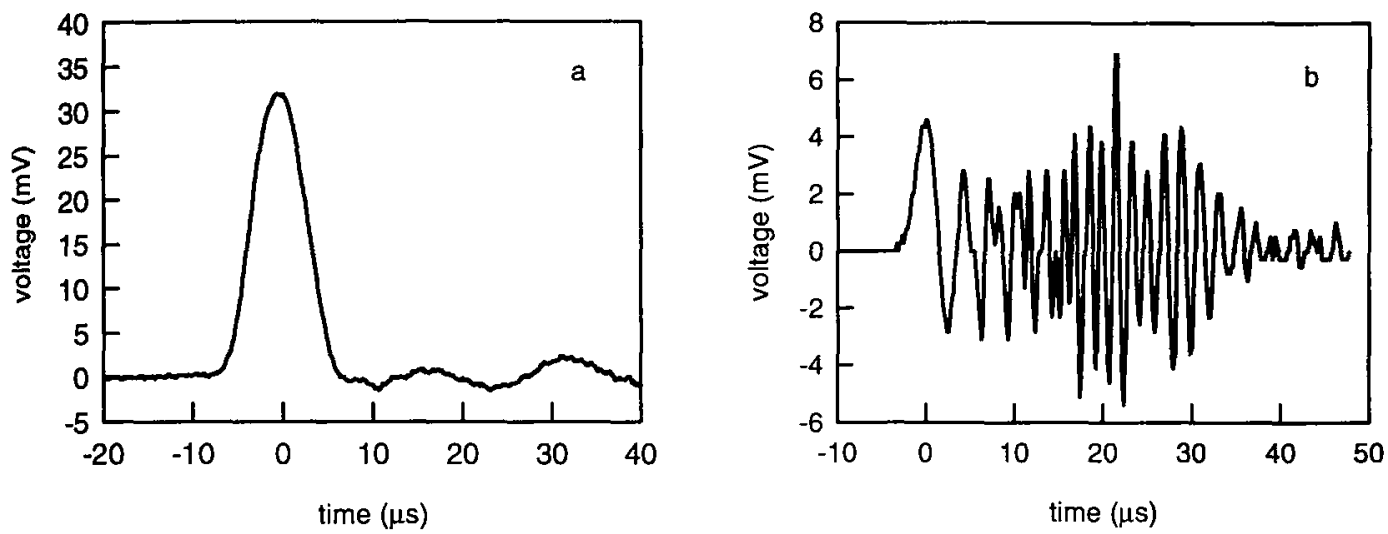

Figure 3: Force measurement from the elastic impact of spheres. (a) $4 \mathrm{~mm}$ diameter; (b) $0.6 \mathrm{~mm}$ diameter.

The magnitude and phase spectra for each of signals of Figure 3 are presented in Figure 4. Again, the frequency axis corresponds to a maximum value of $1.953 \mathrm{MHz}$. The magnitude spectra show much more irregular structures than the ideal pulse does. In the case of the larger sphere, there are two well defined minima within the first 30 components, but then the magnitudes reach the level of the underlying noise and become essentially random. The spectrum from the smaller sphere is above the noise level until a much higher frequency, and the plot shows a number of small peaks and minima up to at least component 160 when the noise begins to be significant.

The phase spectra have been subjected to the numerical procedure known as unwrapping, in which, because phase values are periodic in $2 \pi$, an appropriate number of $2 \pi$ increments are added to each calculated value to yield a monotonic change of phase with frequency. Without unwrapping, the arctan function used to calculate the phase returns values within the interval of 0 to $2 \pi$ and the shape of the characteristic is lost.

The unwrapped phase spectra from each size of sphere impact display a similar shape of curve, but with individual differences of detail. In fact, close comparison of the phase spectra in Figure 4 and those from intermediate sizes of sphere shows that the main differences are steps of $\pi$ or of $2 \pi$. The $2 \pi$ steps represent inaccuracies in the unwrapping process, and they can be removed individually if necessary. The smaller steps of about $\pi$ represent the phase information which defines the pulse shape, as in the ideal case of Figure 1 .

The theoretical pulse of Figure 1 has a phase value of $\pi$ up to the first step. Assuming that the experimental sphere impact force follows a similar, symmetrical form, then any deviation of phase away from $\pi$ in the early part of the record results from the dispersive propagation in the bar. Hence, in principle, the phase characteristics of the bar are given directly by the measured phase spectra up to the first step. Cancellation of dispersion is achieved simply by deriving an accurate representation of this underlying phase characteristic and subtracting it from the phase spectra of measured signals.

In Figure 4(a) both the magnitude and phase spectra for the $4 \mathrm{~mm}$ sphere clearly show the first step to be at component 16. The smaller sphere in Figure 4(b) shows a much more complicated magnitude spectrum in which the minima are not clearly defined, but the phase spectrum shows a very clear $\pi$ step at component 118. Further work with various forms of model pulse have shown that the frequency of the first step is inversely proportional to the pulse width, although the position of other steps depends on the exact pulse shape. This supports the observation that the positions of the first minima for the $4 \mathrm{~mm}$ and $0.6 \mathrm{~mm}$ spheres are approximately at points 16 and 118 respectively. Therefore, the first 118 components of the 0.6 $\mathrm{mm}$ sphere phase spectrum should directly represent the dispersive phase shift of the bar, and higher components can be used if $\pi$ is subtracted from them. In practice the measured phase data needs to be adjusted slightly, and this is described in the following section. 
a
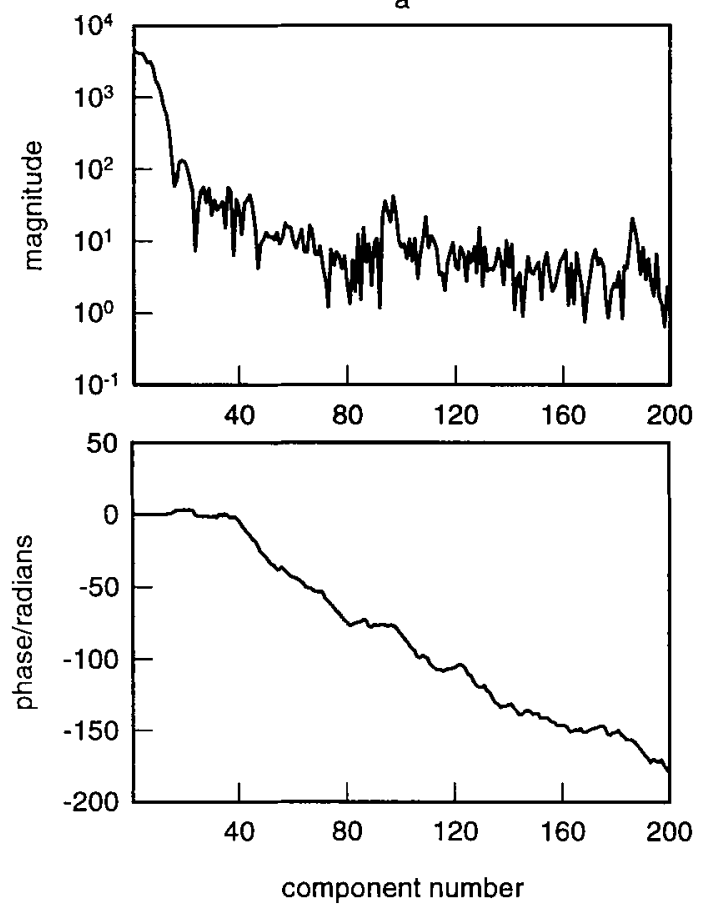

b
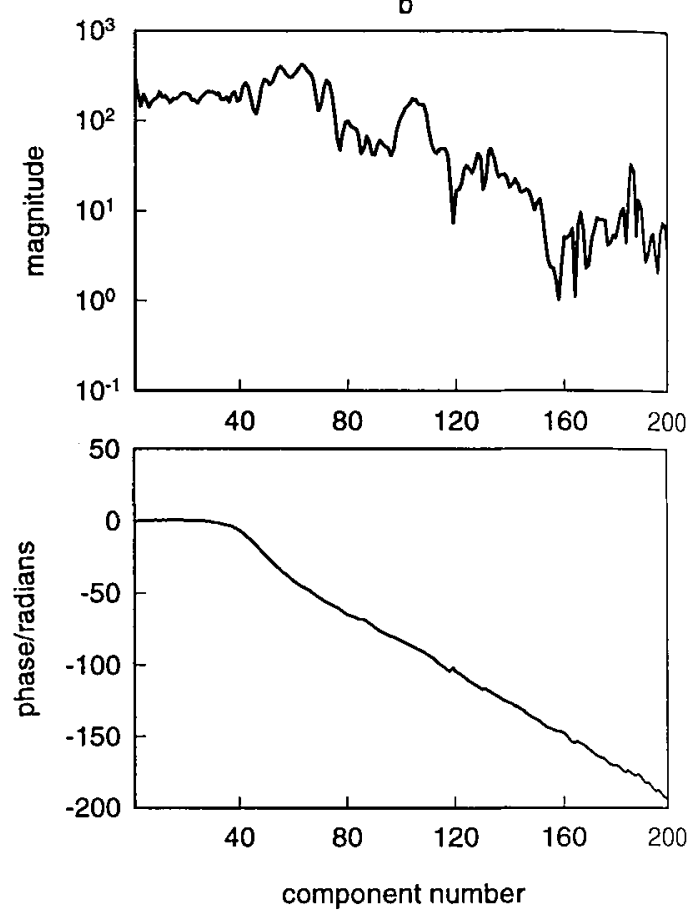

Figure 4: Phase and magnitude spectra, (a) from the $4 \mathrm{~mm}$ sphere impact signal of Figure 3(a); (b) from the $0.6 \mathrm{~mm}$ sphere impact signal of Figure 3(b)

\section{DISPERSION CORRECTION WITH EMPIRICAL PHASE DATA}

If the raw phase data from the $0.6 \mathrm{~mm}$ pulse is simply subtracted from an SHPB signal, then the dispersion correction is not very successful. Close examination of the phase spectra from small particles often reveals the presence of significant oscillations in the first few phase values, which can have a large effect on the reconstructed waveform and are responsible for the poor correction. These are probably the result of aliasing, in which contributions from higher frequency components are added to these low frequency values due to the process of sampling. Some of this arises from noise internally generated in the signal recorder, and so cannot be completely avoided by restricting the signal bandwidth. Hence to achieve successful correction of dispersion, the oscillations need to be removed from the phase spectrum.

Three procedures have been used to derive good quality phase data from the measured signals of small sphere impacts. First, if the oscillations in the phase characteristic are limited to a few values, then these can be manually edited to an appropriate average value. Second, it is found that problems of noise or aliasing affect different parts of the phase spectra from different sized spheres. Hence a hybrid curve can be constructed by joining clean sections of two or more sets of data. Third, an appropriate curve fit can smooth irregularities very effectively. As an example, equation (1) was used to fit the first 50 points of phase data from the $0.6 \mathrm{~mm}$ sphere impact,

$$
\varphi=\frac{a_{1}}{\left(1+e^{\left(a_{2} \times\left(n-a_{3}\right)\right)}\right)^{a_{4}}}+a_{5}+a_{6} \times n
$$

where $\varphi$ is the phase deviation, $n$ is the component number and $a_{1}$ to $a_{5}$ are constants. The values for this tested bar are $a_{1}=76.61, a_{2}=0.1406, a_{3}=53.28, a_{4}=0.9386, a_{5}=76.63$ and $a_{6}=0.0693$. A fit over 50 points was enough to cover the frequency content of measured signals in our SHPB system.

An example of an experimental signal corrected with this data is shown in Figure 5. This is the stress pulse from the collinear impact of a short rod on a long, $6.7 \mathrm{~mm}$ diameter pressure bar, recorded at it position about $100 \mathrm{~mm}$ from the impact end. The dotted trace is the measured signal, where frequency dispersion has caused the rise time to be lengthened and significant oscillations to be added to the 
approximately flat-topped loading pulse. The phase data represented by equation 1 has been subtracted from the signal spectrum, to give the corrected waveform shown by the full line. The oscillations have been very considerably reduced, and the rise time decreased. Note, however, the spurious oscillations which have been added just before the main rise, which are a characteristic of this type of correction.

Figure 6 shows the head of this pulse corrected by the present empirical method, compared with that using the theoretical phase characteristics of the bar [3]. The older method does not work well on this type of signal, and although the oscillations are reduced by a factor of 2 , the correction is very much better with the new method.

Another example, shown in Figure 7, is from a high strain rate material test using the $6.7 \mathrm{~mm}$ SHPB system. The specimen was pure copper, $4.7 \mathrm{~mm}$ diameter, and the average strain rate was $2.5 \times 10^{3} \mathrm{~s}^{-1}$. Dispersion in the loading pulse was minimised experimentally by attaching a double layer of $80 \mathrm{~g} \mathrm{~m}^{-2}$ paper to the impact face of the incident bar. This limits the rise time of the load, and the incident pulse showed virtually no evidence of oscillations. The stress-stain relations obtained from the raw signals and from those corrected by the new empirical technique described above are presented by broken and solid lines respectively. Although oscillations are not very prominent in either stress-strain curve, the correction has introduced a significant difference between them, especially within $4 \%$ of strain. This result indicates that the dispersion of stress pulses can have a significant effect on the stress-strain relations derived from SHPB tests, even if dispersive oscillations are not apparent in the measured signals.

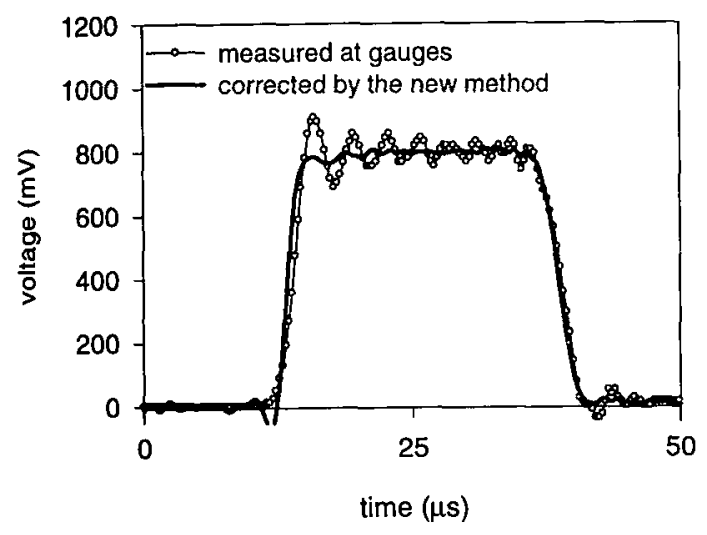

Figure 5: Measured signal from the axial impact of a short rod, corrected by the empirical method.

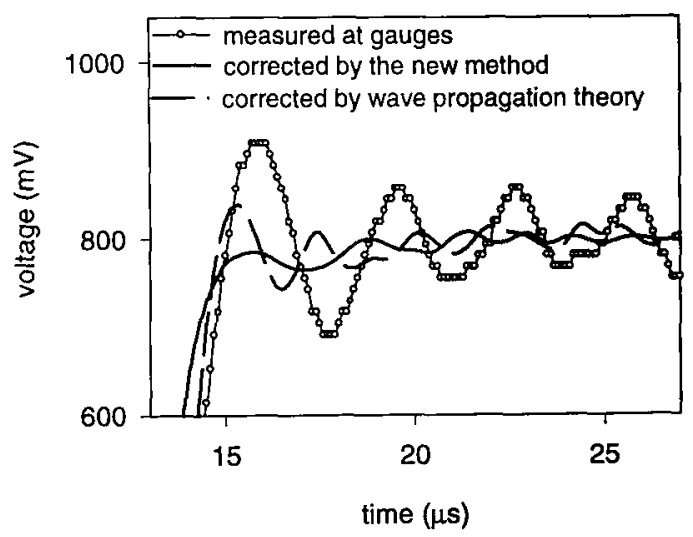

Figure 6: A comparison of the present empirical dispersion technique with the method of Gorham [3] based on the theoretical characteristics of the bar.

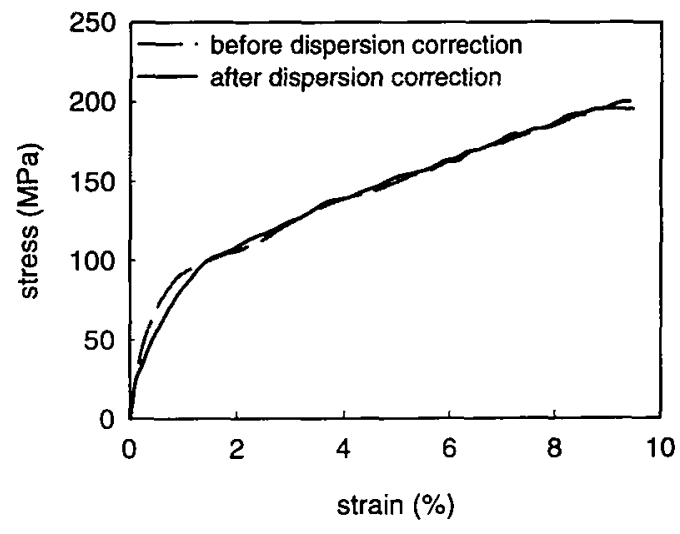

Figure 7: Stress strain curves for copper at a strain rate of $2.5 \times 10^{3} \mathrm{~s}^{-1}$, with and without the empirical dispersion correction. 


\section{DISCUSSION}

This work has shown that large phase values, known to a comparatively high accuracy, are required for a reliable correction for dispersion in pressure bar signals. Most previous work on dispersion correction in bars has been based on a theoretical bar response, and it is not surprising that mismatch between the idealised models and the experimental situation leads to corrections of limited accuracy. A measured phase characteristic automatically takes into account the precise mechanical arrangement of the test, as well as including any dispersive effects from the electronic circuitry. Any change in mode of propagation is also included automatically. The accuracy of the correction is affected by the resolution of the digitisation and by noise, and improvements in these areas would lead to better corrections.

The most accurate phase response is obtained from impacts which are as close to elastic conditions as possible, as this gives a symmetrical pulse and hence a simple ideal phase characteristic. Spheres must be small to allow a sufficient number of phase data points to be obtained, but large enough to give low noise signals.

\section{CONCLUSION}

An accurate, measured dispersion curve for a pressure bar has been derived from the phase characteristics of small sphere impact stress. Dispersion correction of SHPB signals using this empirical curve gives much better results than techniques using wave propagation theory. The new dispersion correction method can effectively and routinely improve the accuracy of stress-strain relations derived from SHPB tests, even when dispersive effects are not prominent in the measured signals.

\section{Acknowledgements}

This work was jointly funded by DRA and the EPSRC Materials Programme. J. Butler and C. Bluck are thanked for their technical support.

\section{References}

[1] Lindholm U.S., J. Mech. Phys. Solids 12 (1964) 317-335.

[2] Hsieh D.Y. and Kolsky H., Proc. Phys. Soc. 71 (1958) 608-612.

[3] Gorham D.A., J Phys. E: Sci Instrum 16 (1983) 477-479.

[4] Follansbee P.S. and Frantz C.E. ASME J Eng. Mat. \& Tech. 105 (1983) 61-66

[5] Gong J.C., Malvern L.E. and Jenkins D.A., ASME J Eng. Mat. \& Tech. 112 (1990) 309-314.

[6] Gary G., Klepaczko J.R., Zhao H., J. Physique IV 1 (1991) C3 403-410.

[7] Safford N.A., Proc. 2nd Int. Sym. on Intense Dynamic Loading and its Effects, Chengdu, China, 1992 378-383.

[8] Lee C.K.B. and Crawford R.C., Meas. Sci. Technol. 4 (1993) 931-937.

[9] Lifshitz J.M. and Leber H., Int. J. Impact Engng. 15 (1994) 723-733.

[10] Lee, C.K.B., Crawford R.C., Mann K.A., Coleman P. and Peterson C., Meas. Sci. Technol. 6 (1995) 853-859.

[11] Gorham D.A. and Wu X.J., Meas. Sci. Technol. 7 (1996) 1227-1232. 